E. D. Wildschut

M. N. Hanekamp

N. J. Vet

R. J. Houmes

M. J. Ahsman

R. A. A. Mathot

S. N. de Wildt

D. Tibboel

\section{Feasibility of sedation and analgesia interruption following cannulation in neonates on extracorporeal membrane oxygenation}

Received: 2 November 2009

Accepted: 22 April 2010

Published online: 28 May 2010

(C) The Author(s) 2010. This article is published with open access at Springerlink.com
E. D. Wildschut · M. N. Hanekamp ·

R. J. Houmes - S. N. de Wildt .

D. Tibboel (®)

Intensive Care and Department of Pediatric Surgery, Erasmus MC-Sophia Children's

Hospital, Dr Molewaterplein 60,

3000 CB Rotterdam, The Netherlands

e-mail: d.tibboel@erasmusmc.nl

Tel.: +31-10-7040704

Fax: +31-10-7036822

\section{N. J. Vet}

Intensive Care and Department of Pediatrics, Erasmus MC-Sophia Children's Hospital, Dr Molewaterplein 60, 3000 CB Rotterdam, The Netherlands

M. J. Ahsman - R. A. A. Mathot Clinical Pharmacology Unit, Department of Hospital Pharmacy, Erasmus MC, Rotterdam, The Netherlands
Abstract Purpose: In most extracorporeal membrane oxygenation (ECMO) centers patients are heavily sedated to prevent accidental decannulation and bleeding complications. In ventilated adults not on ECMO, daily sedation interruption protocols improve short- and longterm outcome. This study aims to evaluate safety and feasibility of sedation interruption following cannulation in neonates on ECMO.

Methods: Prospective observational study in 20 neonates (0.17-5.8 days of age) admitted for ECMO treatment. Midazolam $(n=20)$ and morphine $(n=18)$ infusions were discontinued within $30 \mathrm{~min}$ after cannulation. Pain and sedation were regularly assessed using COMFORT$\mathrm{B}$ and visual analog scale (VAS) scores. Midazolam and/or morphine were restarted and titrated according to protocolized treatment algorithms. Results: Median (interquartile range, IQR) time without any sedatives was $10.3 \mathrm{~h}$ (5.0-24.1 h). Median interruption duration for midazolam was $16.5 \mathrm{~h}$ (6.6-29.6 h), and for morphine was $11.2 \mathrm{~h}(6.7-39.4 \mathrm{~h})$. During this period no accidental extubations, decannulations or bleeding complications occurred.
Conclusions: This is the first study to show that interruption of sedatives and analgesics following cannulation in neonates on ECMO is safe and feasible. Interruption times are 2-3 times longer than reported for adult ICU patients not on ECMO. Further trials are needed to substantiate these findings and evaluate short- and long-term outcomes.

Keywords Neonate .

Extracorporeal membrane oxygenation - Sedation · Daily interruption

\section{Abbreviations}

\begin{tabular}{|c|c|}
\hline CS & COMFORT-B scale \\
\hline ECMO & $\begin{array}{l}\text { Extracorporeal } \\
\text { membrane oxygenation }\end{array}$ \\
\hline $\mathrm{ICU}$ & Intensive care unit \\
\hline IQR & Interquartile range \\
\hline SNAP II & $\begin{array}{l}\text { Score for Neonatal } \\
\text { Acute Physiology } \\
\text { Version II }\end{array}$ \\
\hline SNAPPE II & $\begin{array}{l}\text { Score for Neonatal } \\
\text { Acute Physiology, } \\
\text { Perinatal Extension, } \\
\text { Version II }\end{array}$ \\
\hline AS & Visual analog scale \\
\hline
\end{tabular}




\section{Introduction}

Extracorporeal membrane oxygenation (ECMO) is a form of cardiopulmonary bypass for patients with pulmonary or circulatory failure unresponsive to conventional treatment. Most patients are heavily sedated to prevent accidental decannulation or impeded ECMO flow due to movement or suboptimal cannula position. However, prolonged and high cumulative doses of opioids and benzodiazepines have been associated with tolerance, physical dependency, and consequently withdrawal syndrome in neonates and children [1-5]. Sedation guidelines for pediatric ICU patients have been developed but are in part hampered by the lack of high-quality evidence, and exclude neonates and ECMO patients $[6,7]$. Strategies to decrease cumulative doses and duration of continuous infusions with the intent to reduce consequent adverse events include daily interruption or even complete withholding of continuous sedation $[8,9]$. The latter strategies were both shown to significantly reduce total cumulative doses of sedative drugs without an increase in complications. More importantly, ventilator-free days, length of ICU stay, and occurrence of posttraumatic stress syndrome were also significantly reduced [10-12]. Two meeting reports on daily interruption in children presented a reduction of midazolam dose in the intervention group; both studies lacked power to show an effect on mechanical ventilation or ICU stay $[13,14]$. To our knowledge, there are no such data on neonates or children on ECMO support.

The aim of our study is therefore to evaluate safety and feasibility of initial interruption of analgesia and sedatives in neonates following cannulation for ECMO.

\section{Methods}

Study design and setting

Prospective observational cohort study at a designated ECMO center.

The institutional medical ethics review board approved the study, and informed consent was obtained from the parents or legal representatives.

Patients

All neonates $<7$ days old admitted for ECMO in 1 year were eligible for enrolment.

Criteria for ECMO treatment were: gestational age $>34$ weeks, birth weight $>2.0 \mathrm{~kg}$, mechanical ventilation $<7$ days, alveolar arterial oxygen difference $>600 \mathrm{mmHg}$, and oxygenation index $>25$ for more than $6 \mathrm{~h}$.

All received standardized anesthesia during cannulation, consisting of fentanyl $5 \mathrm{mcg} / \mathrm{kg}$ bolus injection, morphine $50 \mathrm{mcg} / \mathrm{kg} / \mathrm{u}$, and midazolam $200 \mathrm{mcg} / \mathrm{kg} / \mathrm{u}$ continuous infusion during cannulation. On ICU admission, severity of illness was assessed using the Score for Neonatal Acute Physiology Version II (SNAP II) and the Score for Neonatal Acute Physiology, Perinatal Extension, Version II (SNAPPE II).

\section{Sedation and analgesia assessment}

Pain and sedation were routinely assessed by the attending nurse using validated pain (visual analog scale, VAS) and sedation (COMFORT-B) scores [15, 16]. Morphine and midazolam infusions were discontinued 30-60 min after cannulation and resumed on the guidance of regularly determined CS and VAS scores.

When COMFORT-B score was 17 or higher, continuous midazolam $100 \mathrm{mcg} / \mathrm{kg} / \mathrm{h}$ was started after a loading dose of $200 \mathrm{mcg} / \mathrm{kg}$. Morphine $10 \mathrm{mcg} / \mathrm{kg} / \mathrm{u}$ was started after a loading dose of $100 \mathrm{mcg} / \mathrm{kg}$ when VAS score was 4 or higher, or when sedation was ineffective with midazolam $(>300 \mathrm{mcg} / \mathrm{kg} / \mathrm{u})$, or at the discretion of the attending medical team. Fentanyl was used for procedural analgesia or as rescue medication.

\section{Outcome parameters}

Primary: duration of sedation interruption

Secondary: need for rescue medication and number of complications (extubations, decannulation, impairment of ECMO flow by $50 \%$ )

\section{Statistical analysis}

All values are presented as median (IQR) unless otherwise indicated. Groups were compared using MannWhitney test. For correlation analyses the Spearman signed-rank test was used. A $p$ value $<0.05$ was considered significant. All statistical analyses were performed using Graphpad Prism v4 (Graphpad Software Inc., La Jolla CA, USA).

\section{Results}

Patient characteristics

Twenty-seven patients received ECMO support during the study period. Twenty-one met the inclusion criterion, but one of them died within $24 \mathrm{~h}$ after cannulation. So, the analysis included 20 patients. Median postnatal age (range) was $0.79(0.17-5.8)$ days (Table 1$)$. All patients received midazolam median (IQR) $110 \mathrm{mcg} / \mathrm{kg} / \mathrm{u}(100-200 \mathrm{mcg} /$ $\mathrm{kg} / \mathrm{u})$ and morphine $10.9 \mathrm{mcg} / \mathrm{kg} / \mathrm{u} \quad(10-20 \mathrm{mcg} / \mathrm{kg} / \mathrm{u})$ 
Table 1 Clinical characteristics

\begin{tabular}{|c|c|c|}
\hline Patients $(n)$ & 20 & \\
\hline Female/male $(n)$ & $10 / 10$ & \\
\hline Mortality (\%) & 25 & \\
\hline $\mathrm{CDH}$ & 7 & \\
\hline MAS & 10 & \\
\hline Pneumonia & 1 & \\
\hline Sepsis & 1 & \\
\hline \multirow[t]{2}{*}{ Pulmonary valve atresia } & 1 & \\
\hline & Median (IQR) & (Range) \\
\hline SNAP II & $16(16-23)$ & $(26-35)$ \\
\hline SNAPPE II & $33(16-34)$ & $(16-54)$ \\
\hline Oxygenation index prior to ECMO & 38 & $(21-54)$ \\
\hline $\mathrm{AaDO}_{2}$ prior to $\mathrm{ECMO}(\mathrm{mmHg})$ & 599 & $(522-624)$ \\
\hline Age (days) & $0.79(0.29-3.4)$ & $(0.17-6.8)$ \\
\hline Length of ECMO (h) & $123(88-218)$ & $(53-462)$ \\
\hline Gestational age (weeks) & $401 / 7(38$ 1/7-41 4/7) & $(35 / 5 / 7-423 / 7)$ \\
\hline Birth weight (kg) & $3.1(2.8-3.6)$ & $(2.3-4.0)$ \\
\hline Morphine dose pre-ECMO (mcg/kg/u) & $10.9(10-20)$ & $(8.8-33)$ \\
\hline Midazolam dose pre-ECMO (mcg/kg/u) & $110(100-200)$ & $(50-220)$ \\
\hline
\end{tabular}

Data given are number of patients or median values (IQR) (range)

$C D H$ congenital diaphragmatic hernia, $M A S$ meconium aspiration syndrome, $\mathrm{AaDO}_{2}$ arterial alveolar oxygen difference

before cannulation. Five received phenobarbital for suspected convulsions prior to cannulation for ECMO. Twelve patients received vecuronium bromide prior to ECMO. All patients received inotropic support and antibiotics.

\section{Sedation interruption}

Midazolam was discontinued in all 20 patients; morphine in 18. Median interruption time for both drugs combined was $10.3 \mathrm{~h}$ (IQR 5.0-24.1 h). Median interruption time for midazolam was $16.5 \mathrm{~h}$ (IQR 6.6-29.6); median interruption time for morphine was $11.2 \mathrm{~h}$ (IQR 6.739.4 h) (Fig. 1). Interruption times were shorter for patients with higher cumulative doses of midazolam or morphine $(r=-0.54, \quad p=0.013$ and $r=-0.58$, $p=0.008$, respectively).

Interruption times for patients with meconium aspiration syndrome were shorter than for patients with other diagnoses; the difference did not reach statistical significance [6.8 h (3.2-15.2 h) versus $16.0 \mathrm{~h}(8.7-35.1 \mathrm{~h}), p=0.07]$.

Duration of interruption did not differ between patients with and without concomitant phenobarbital use, male and female patients, or survivors and nonsurvivors. There was no significant correlation between SNAP II or SNAPPE II score and interruption time.

\section{Safety}

No accidental decannulations or extubations during sedation interruption were observed, neither agitation resulting in impairment of ECMO flow nor accidental bleeding.
Rescue medication

Three patients $(15 \%)$ received fentanyl during the interruption period, one for perceived discomfort and two for procedural analgesia.

Level of sedation

Both CS and VAS scores were low during sedation interruption (Table 2). In seven patients (35\%) midazolam or morphine was restarted on the guidance of

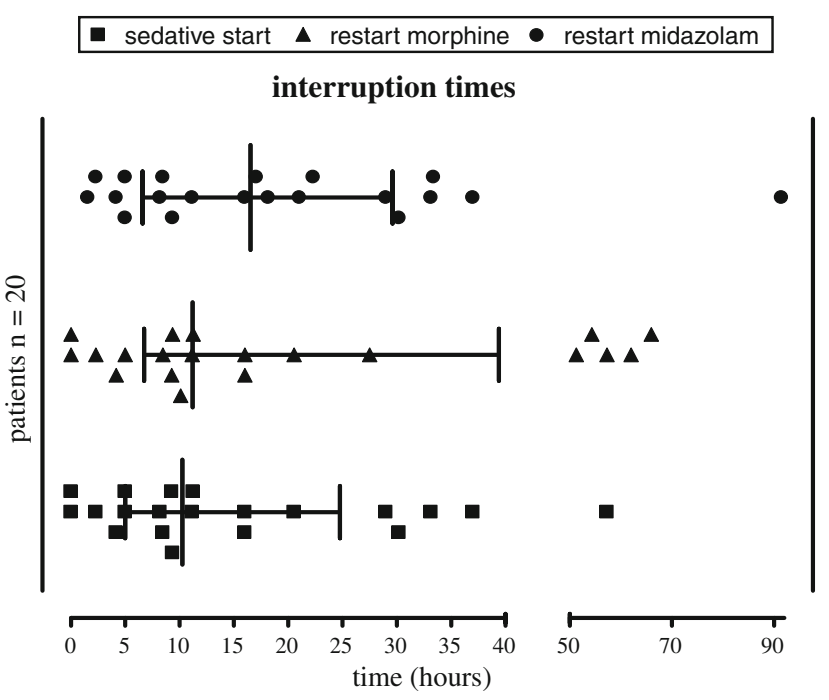

Fig. 1 Individual interruption times of midazolam, morphine, and of both sedatives. Each dot represents one patient 
Table 2 Interruption duration, plasma levels, CS, and VAS at restart of medication

\begin{tabular}{llll}
\hline & Median & IQR & Range \\
\hline Interruption duration $(\mathrm{h})$ & $10: 25$ & $5: 00-24: 10$ & $0: 00-57: 30$ \\
Median CS during cessation of medication & 8.5 & $8-9$ & $7-15$ \\
VAS & 1 & $0-2$ & $0-5$ \\
CS at restart of medication $(n=13)$ & 17 & $11-18$ & $10-21$ \\
\hline
\end{tabular}

COMFORT-B score $>17$. In the other 13 patients either no CS was recorded at the moment of restart of medication $(n=7)$ or midazolam or morphine was started despite COMFORT-B or VAS score below the cutoff point $(n=6)$. Reasons for start of medication were: perceived discomfort manifesting as unexplained cardiovascular or respiratory instability, or suspected discomfort in anticipation of a medical procedure.

\section{Discussion}

To our knowledge this is the first study that shows that prolonged interruption of sedatives and analgesics is feasible in neonates on ECMO. Interruption of morphine or midazolam did not cause any major complications. On average, patients remained adequately sedated for $10 \mathrm{~h}$ after cessation of medication. This is much longer than reported in adult patients not on ECMO [8].

In the present study median COMFORT-B scores during the study period were low overall: 8.5 (IQR8-9), indicating oversedation [17]. In combination with predominantly low COMFORT-B and VAS scores at time of restart of sedatives, this goes to show that, if anything, sedation was reintroduced early rather than late. In line with adult studies, interruption of medication seems more effective in preventing oversedation than does protocolized sedation alone.

Potentially altered disposition or effect of the drugs given, due to age, pre-existing disease, cannulation or ECMO treatment, could play a role in the longer interruption duration in our patients compared with adults. As all were younger than 7 days, effect of age on interruption duration could not be studied. We could not identify a relation between critical illness scores and interruption duration. Pharmacokinetic data of midazolam in these patients show increased volume of distribution and clearance on ECMO [18]. This suggests that the observed interruption times are not related to increased plasma levels on ECMO.

We did find a shorter interruption duration in patients with high cumulative doses of morphine and midazolam prior to ECMO treatment, which could imply increased tolerance to both drugs.

As a limitation of the study, there was a high percentage of protocol violation. In $60 \%$ of cases morphine was started prior or simultaneously with midazolam; in $10 \%$ morphine was never discontinued. In all but one patient VAS scores were below 4, indicating no pain at time of morphine restart. Morphine was mostly used as a sedative, either as first choice or as an addition to midazolam even when midazolam dose was below $300 \mathrm{mcg} / \mathrm{kg} / \mathrm{u}$. The attending physician was allowed to deviate from protocol based on clinical assessment of the patient. In many neonatal ICUs morphine is the sedative of choice in ventilated (pre-)term infants, therefore attending physicians may opt for morphine more easily than for midazolam. Furthermore, procedures perceived as painful may have elicited the choice for morphine as a prophylactic analgesic. Due to these protocol violations it is impossible to discriminate between the sedative effects of midazolam and morphine in this study.

A second limitation lies in either missing or low COMFORT-B scores at restart of medication. In two patients medication was restarted prior to a procedure without scores being performed. In nine patients nurses indicated that the patient was uncomfortable or more awake than deemed necessary, despite low COMFORT-B scores. Ista et al. [17] showed that interpretation of CS scores between 11 and 22 is difficult in children on the ICU and may necessitate an additional score.

Finally, doctors' and nurses' fear of accidental decannulation or ECMO system failures may precipitate earlier restart of sedatives. Our findings suggest that the actual interruption duration could have been longer if protocol compliance had been better.

Mulla et al. [19] reported $100 \%$ adequate sedation with midazolam and morphine in a study cohort of 20 neonates on ECMO; unfortunately, dose adjustments were not reported. Our findings are in line with previous studies showing that, even if patients are oversedated and sedation protocols dictate to lower doses of sedative drug, these dose adjustments are not always made [20].

\section{Conclusions}

Interruption of sedatives and analgesics is feasible and safe in neonates on ECMO without increased risk of complications. Interruption times are 2-3 times longer than reported in adult ICU patients. Future trials are needed to substantiate these findings and evaluate outcome benefits. 
Conflict of interest statement The authors declare that they have no competing interests.

Open Access This article is distributed under the terms of the Creative Commons Attribution Noncommercial License which permits any noncommercial use, distribution, and reproduction in any medium, provided the original author(s) and source are credited.

\section{References}

1. Fonsmark L, Rasmussen YH, Carl P (1999) Occurrence of withdrawal in critically ill sedated children. Crit Care Med 27:196-199

2. Ista E, van Dijk M, Gamel C, Tibboel D, de Hoog M (2008) Withdrawal symptoms in critically ill children after long-term administration of sedatives and/or analgesics: a first evaluation. Crit Care Med 36:2427-2432

3. Suresh S, Anand KJ (2001) Opioid tolerance in neonates: a state-of-the-art review. Paediatr Anaesth 11:511-521

4. Hughes J, Gill A, Leach HJ, Nunn AJ, Billingham I, Ratcliffe J, Thornington R, Choonara I (1994) A prospective study of the adverse effects of midazolam on withdrawal in critically ill children. Acta Paediatr 83:11941199

5. Ista E, van Dijk M, Gamel C, Tibboel D, de Hoog M (2007) Withdrawal symptoms in children after long-term administration of sedatives and/or analgesics: a literature review.

"Assessment remains troublesome". Intensive Care Med 33:1396-1406

6. Playfor S, Jenkins I, Boyles C, Choonara I, Davies G, Haywood T, Hinson G, Mayer A, Morton N, Ralph T, Wolf A (2006) Consensus guidelines on sedation and analgesia in critically ill children. Intensive Care Med 32:1125-1136

7. Prins S, van Dijk M, Tibboel D (2006) Sedation and analgesia in the PICU: many questions remain. Intensive Care Med 32:1103-1105

8. Kress JP, Pohlman AS, O'Connor MF, Hall JB (2000) Daily interruption of sedative infusions in critically ill patients undergoing mechanical ventilation. N Engl J Med 342:14711477
9. Strom T, Martinussen T, Toft P (2010) A protocol of no sedation for critically ill patients receiving mechanical ventilation: a randomised trial. Lancet 375:475-480

10. Carson SS, Kress JP, Rodgers JE, Vinayak A, Campbell-Bright S, Levitt J, Bourdet S, Ivanova A, Henderson AG, Pohlman A, Chang L, Rich PB, Hall J (2006) A randomized trial of intermittent lorazepam versus propofol with daily interruption in mechanically ventilated patients. Crit Care Med 34:1326-1332

11. Kress JP, Gehlbach B, Lacy M, Pliskin N, Pohlman AS, Hall JB (2003) The long-term psychological effects of daily sedative interruption on critically ill patients. Am J Respir Crit Care Med 168:1457-1461

12. Mehta S, Burry L, Martinez-Motta JC, Stewart TE, Hallett D, McDonald E, Clarke F, Macdonald R, Granton J, Matte A, Wong C, Suri A, Cook DJ (2008) A randomized trial of daily awakening in critically ill patients managed with a sedation protocol: a pilot trial. Crit Care Med 36:2092-2099

13. Jayashree M, Gupta VK, Singhi S (2007) Randomized controlled trial of interrupted versus continuous sedative infusions in ventilated children-a pilot study. Pediatr Crit Care Med 8:A182

14. Heesen G, Verlaat C, Pickkers P (2007) Effects of daily interruption of sedatives in critically ill children. Pediatr Crit Care Med 8:A182

15. Bouwmeester NJ, Hop WC, van Dijk M, Anand KJ, van den Anker JN, Tibboel D (2003) Postoperative pain in the neonate: age-related differences in morphine requirements and metabolism. Intensive Care Med 29:2009-2015
16. van Dijk M, de Boer JB, Koot HM, Tibboel D, Passchier J, Duivenvoorden HJ (2000) The reliability and validity of the COMFORT scale as a postoperative pain instrument in 0 to 3-year-old infants. Pain 84:367-377

17. Ista $E$, van Dijk M, Tibboel D, de Hoog M (2005) Assessment of sedation levels in pediatric intensive care patients can be improved by using the COMFORT "behavior" scale. Pediatr Crit Care Med 6:58-63

18. Ahsman MJ, Hanekamp M, Wildschut ED, Tibboel D, Mathot RAA (2010) Population pharmacokinetics of midazolam and metabolites during venoarterial extracorporeal membrane oxygenation in neonates. Clin Pharmacokinet 49(6):407-419

19. Mulla H, Lawson G, Peek GJ, Firmin RK, Upton DR (2003) Plasma concentrations of midazolam in neonates receiving extracorporeal membrane oxygenation. ASAIO J 49:41-47

20. de Wildt SN, de Hoog M, Vinks AA, Joosten KF, van Dijk M, van den Anker JN (2005) Pharmacodynamics of midazolam in pediatric intensive care patients. Ther Drug Monit 27:98-102 